\title{
随机序列的新息方法在电力系统 负荷预报中的应用
}

\author{
徐立子 \\ (电力科学研究院) \\ 杜金观 项静恬顾 岗 \\ (中国科学院数学研究所)
}

电力系统次日 24 小时预报和安全控制所需的负荷预报是电网调度中安全、经济控制的基 础. 据了解, 目前国内外多数电力系统调度为获得负荷预报, 主要依靠有经验的运行方式人员, 通过过去的负荷数据, 人工估计而得. 本文采用随机时间序列方法建立电力系统负荷模型, 并 利用随机序列的新息方法来进行电力系统负荷预报 ${ }^{[1]}$, 得到了较满意的结果. 此法既快速, 所 占存储容量又少, 误差也小, 为电力系统调度安全、经济控制提供了一个预报自动化工具. 文 中并提出, 采用新息协方差作为预报可靠性的判据. 此判据为找出样本中的不合理数据及样 本是否符合模型的一种数学判别法.

\section{一、电力系统负荷模型的建立}

电力系统负荷时间序列 $Y_{1}, Y_{2}, \cdots, Y_{n}, \cdots$ (每小时一个数据)为一非平稳过程, 并有一个 长度为 24 小时的周期. 故将此序列进行差分运算, 作出新时间序列

$$
Z_{t}=\nabla \nabla_{24} Y_{t}=(1-B)\left(1-B^{24}\right) Y_{t},
$$

对此新序列作为平稳过程处理.

对我国某两电力系统进行分析计算认为, 可采用 25 阶乘积形式的疏系数模型来表示电力 系统的负荷模型,如 (2) 式:

$$
Z_{t}=\nabla \nabla_{24} Y_{t}=\left(1-\theta_{1} B\right)\left(1-\theta_{24} B^{24}\right) a_{t}=\left(1-\theta_{1} B-\theta_{24} B^{24}+\theta_{25} B^{25}\right) a_{t},
$$

式中 $a_{t}$ 为白噪声.

模型参数估计可采用极大似然估计求得. 为了使负荷预报程序简单且快速, 所占存储容 量少, 误差也能满足要求, 以适应电网调度在线控制机的需要, 本文对模型 (2) 采用样本协方 差直接进行预报.

\section{二、随机序列的新息预报与预报可菲性判据}

1. 设观测序列 $Z$ 、为平稳序列

模型

$$
\Phi(B) Z_{t}=\theta(B) a_{t},
$$

令 $W_{\imath}=\Phi(B) Z_{\imath}$, 则模型为:

可证明新息

$$
W_{t}=\theta(B) a_{i}
$$

本文 1979 年 12 月 26 日收到。 
为一白噪声过程.

$$
\epsilon_{t}=Z_{t}-\hat{Z}_{t \mid t-1}=W_{t}-\hat{W}_{t \mid t-1}
$$

2. 由文献 [1], 导出滑动平均模型的适时预报公式如下:

新息

$$
Z_{t}=\nabla \nabla_{24} Y_{t},
$$

\section{和息}

$$
e_{k}= \begin{cases}Z_{1}, & \text { 当 } k=1, \\ Z_{k}-\sum_{j=1}^{k-1} J_{k i} e_{i}, & \text { 当 } 1<k \leqslant q, \\ Z_{k}-\sum_{i=k-q}^{k-1} J_{k i} e_{i}, & \text { 当 } k>q,\end{cases}
$$

其中

$$
J_{k j}=\left\{\begin{array}{l}
{\left[R_{k j}^{Z}-\sum_{i=1}^{j-1} J_{k i} J_{i i} R_{i i}^{e}\right] \quad\left[R_{i j}^{\epsilon_{j}}\right]^{-1}, \text { 当 } 1 \leqslant k \leqslant q, 1 \leqslant i \leqslant k-1,} \\
{\left[R_{k i}^{Z}-\sum_{i=k-q}^{j-1} j_{k i} J_{i i} R_{i i}^{e}\right] \quad\left[R_{i j}^{e}\right]^{-1}, \text { 当 } k>q, k-q \leqslant j \leqslant k-1 .}
\end{array}\right.
$$

$R_{k j}^{Z}=E Z_{k} Z_{i}$, 具体计算时由样本协方差代替.

$$
R_{k k}^{e}= \begin{cases}R_{11}^{Z}, & \text { 当 } k=1, \\ R_{k k}^{Z}-\sum_{j=1}^{k-1} J_{k i}^{2} R_{i j}^{e}, & \text { 当 } 1<k \leqslant q, \\ R_{k k}^{Z}-\sum_{j=k-q}^{k-1} J_{k i}^{2} R_{i j}^{e}, & \text { 当 } k>q .\end{cases}
$$
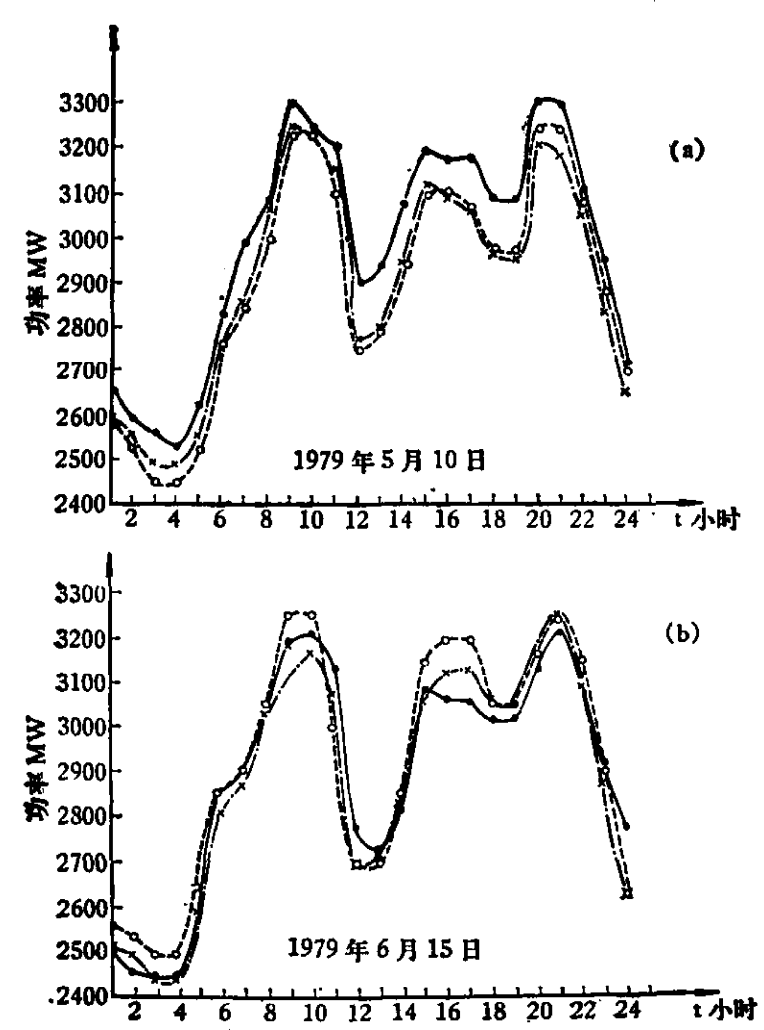
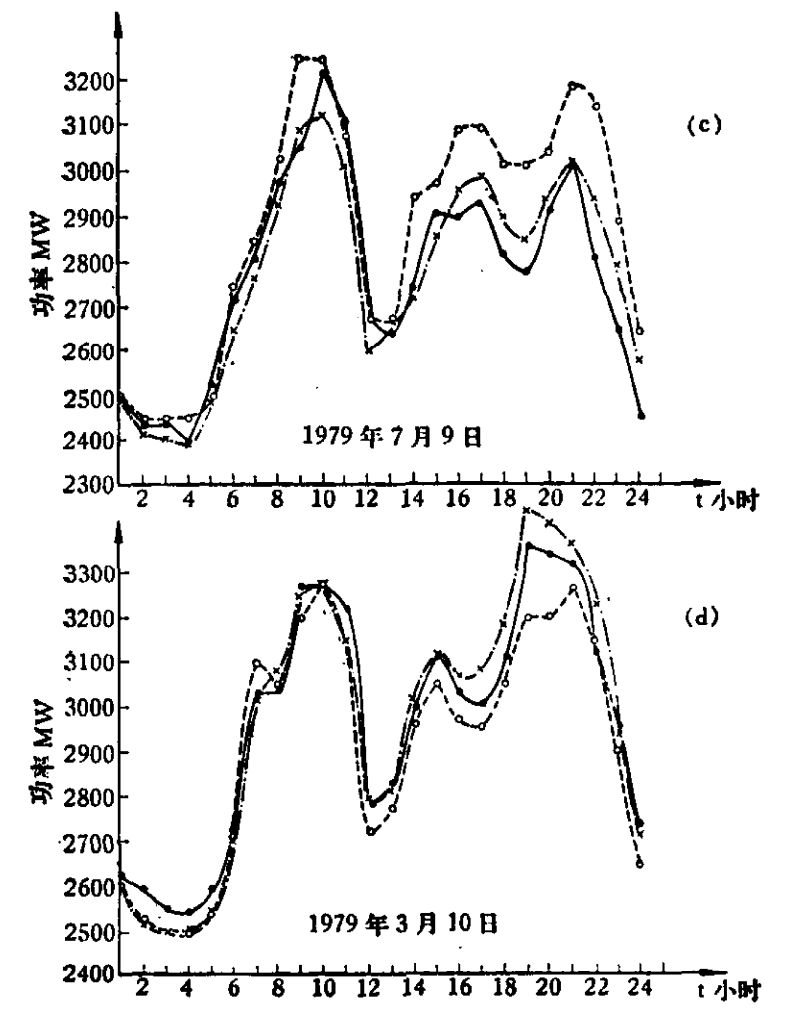

图 1 某电力系统负荷实测值与新息法预报和人工预报的比较

- - ・实测; $x-.-x$ 新息预报; 0 - 0 人工预报 
则 $l$ 步预报公式为

$$
\hat{Z}_{k+l l k}= \begin{cases}\sum_{j=1}^{k} J_{k+l, j} e_{j}, & \text { 当 } k+l \leqslant q, \\ \sum_{j=k+l-q}^{k} J_{k+l}, e_{i}, & \text { 当 } k+l>q .\end{cases}
$$

\section{3. 预报可靠性判据}

由于新息序列为白噪声序列，故新息协方差 $R_{k k}^{e}$ 可作为预报可靠性和准确性的判据. 正 ，常情况下样本新息序列的协方差序列应该是稳定的. 若样本中出现不合理数据(如机组、线路 等突然故障, 强制性拉负荷, 大修,做试验, 气象突变引起负荷突变等),或者样本不符合模型 时,则样本新息序列的协方差会出现较大的起伏.

\section{三、具体电网计筆结果}

对我国某电力系统进行负荷预报的结果示于图 1. 图中并将负荷实测值与人工预报结果 同时示出,以便比较。

本文提出的新息法负荷预报程序在电力科学研究院 6912 数字机上进行数值计算,机器平 均速度为 15 万次/秒。程序用 BASIC 语言编写. 负荷样本采用 7 天 168 个数据, 为进行差分 共需 193 个数据. 次日 24 小时预报包括打印出样本数据、差分值、判据及预报结果, 总共 2.5 分钟. 在正常情况下 24 小时预报的相对误差为 2-4\%, 故可代替人工预报（在国内人工预报 一般需 2 小时). 程序所占存储容量少. 从建立模型到预报包括中间工作单元、原始数据存放 单元等总共只需 $1 \mathrm{~K}$ 单元. 用同一程序并可进行电力系统在线安全控制所需的半小时 -2 小 时预报, 相对误差在 $2 \%$ 以内. 故数字机用新息法进行负荷预报, 将可能代替人工劳动, 而成 为电网数字机安全、经济控制中的重要一环.

[1] 杜金观、潘一民,数学学报, 20 (1977), 16-27.

[2] Aasnaes, H. B., Kailath, T., IEEE, AC-18(1973), 601-607. 